\title{
Complete Controllability for Fractional Evolution Equations
}

\author{
Xia Yang ${ }^{1}$ and Haibo $\mathrm{Gu}^{2,3}$ \\ ${ }^{1}$ School of Science, Shihezi University, Shihezi, Xinjiang 832003, China \\ ${ }^{2}$ School of Mathematics Sciences, Xinjiang Normal University, Urumqi, Xinjiang 830054, China \\ ${ }^{3}$ School of Mathematics and Computational Science, Xiangtan University, Xiangtan, Hunan 411105, China
}

Correspondence should be addressed to Haibo Gu; hbgu_math@163.com

Received 3 December 2013; Revised 13 March 2014; Accepted 20 March 2014; Published 10 April 2014

Academic Editor: Simeon Reich

Copyright (C) 2014 X. Yang and H. Gu. This is an open access article distributed under the Creative Commons Attribution License, which permits unrestricted use, distribution, and reproduction in any medium, provided the original work is properly cited.

\begin{abstract}
The paper is concerned with the complete controllability of fractional evolution equation with nonlocal condition by using a more general concept for mild solution. By contraction fixed point theorem and Krasnoselskii's fixed point theorem, we obtain some sufficient conditions to ensure the complete controllability. Our obtained results are more general to known results.
\end{abstract}

\section{Introduction}

Fractional differential equations have recently been proved to be valuable tools in the modeling of many phenomena in various fields of science and engineering. It draws a great application in nonlinear oscillations of earthquakes and many physical phenomena such as seepage flow in porous media and in fluid dynamic traffic model. There has been a significant development in fractional differential equations in recent years, see the monographs of Kilbas et al. [1], Miller and Ross [2], Podlubny [3], Lakshmikantham et al. [4], and the papers [5-14] and the references therein.

Some recent papers investigated the problem of the existence of a mild solution for abstract differential equation with fractional derivative [15-23]. However, the results in $[15,16,18,19]$ are incorrect since the considered variation of constant formulas is not appropriate [17]. Zhou and Jiao $[22,23]$ introduced two characteristic solution operators and gave a suitable concept on a mild solution by applying Laplace transform and probability density functions. But the condition that the analytic semigroup $\{T(t)\}_{t \geq 0}$ was uniformly bounded was too strong. Shu et al. [20] researched the existence of mild solutions for impulsive fractional partial differential equation. But, Fečkan et al. [24] had pointed out that the definition of solution of impulsive fractional differential equation was not correct. By using Laplace transform, Shu and Wang [25] gave a definition of mild solution for fractional differential equation with order $1<\alpha<2$ and investigated its existence. Agarwal et al. [26] studied the existence and dimension of the set for mild solutions of semilinear fractional differential equations inclusions.

In 1960, Kalman first introduced the concept of controllability which leads to some very important results regarding the behavior of linear and nonlinear dynamical systems. There are various works of complete controllability of systems represented by differential equations, integrodifferential equations, differential inclusions, neutral functional differential equations, and impulsive differential inclusions in Banach spaces (see $[8,27-29]$ and the references therein). Recently, more and more researchers also pay attention to study the controllability of fractional order evolution systems (see [21, $30,31]$ and the references and therein). Unfortunately, the concept of mild solutions used in $[30,31]$ was not suitable for fractional evolution systems at all and the corresponding definition of mild solutions is only a simple extension of the mild solutions of integer order systems. Wang and Zhou [21] investigated the complete controllability of fractional evolution systems with two characteristic solution operators introduced by them.

The nonlocal condition can be applied in physics with better effect than the classical initial condition $x(0)=x_{0}$. Nonlocal condition was initiated by Byszewski [32] when he proved the existence and uniqueness of mild and classical solutions of nonlocal Cauchy problems. As remarked by Byszewski and Lakshmikantham [33], the nonlocal condition can be more useful than the standard initial condition to describe some physical phenomena. 
Inspired by the above discussions, in this paper, we consider a class of fractional evolution equations. By using a more general definition of mild solution, we obtain some sufficient conditions to ensure the complete controllability.

We consider the following fractional evolution equations:

$$
\begin{gathered}
{ }^{C} D_{t}^{\alpha} x(t)=A x(t)+f(t, x(t))+B u(t), \quad t \in J=[0, b], \\
x(0)+g(x)=x_{0},
\end{gathered}
$$

where ${ }^{C} D_{t}^{\alpha}$ is the Caputo fractional derivative of order $\alpha \epsilon$ $(0,1]$, the state $x(\cdot)$ takes value in a Banach space $X$, the control function $u(\cdot)$ is given in $L^{2}(J, U)$, with $U$ as a Banach space, $B$ is a bounded linear operator from $U$ into $X, A$ is a sectorial operator on $X, f: J \times X \rightarrow X$ and $g: X \rightarrow X$ are given functions satisfying some assumptions, and $x_{0} \in X$.

The rest of this paper is organized as follows. In Section 2, some notations and preparations are given. A suitable concept on a mild solution for our problem is introduced. In Section 3, the complete controllability results are obtained by using fixed point theorems. Some conclusions are given in Section 4.

\section{Preliminaries}

In this section, we will firstly introduce fractional integral and derivative, some notations about sectorial operators, solution operators, and analytic solution operators and then give the definition of a mild solution of system (1).

Throughout this paper, $\mathbb{R}, \mathbb{C}$ denote the sets of real and complex numbers, respectively, and $\mathbb{R}_{+}=[0, \infty)$. By $C(J, X)$, we denote the space of all continuous functions from $J$ to $X$. $\mathscr{L}(X)$ is the space of all bounded linear operators from $X$ to $X . D(A)$ denotes domain of $A$, while $\rho(A)$ means resolvent set of $A$ and $R(\lambda, A)=(\lambda I-A)^{-1}$ stands for the resolvent operator of $A$.

Definition 1 (see [3]). The fractional integral of order $p$ with the lower limit $a$ for a function $f:[a, \infty) \rightarrow \mathbb{R}$ is defined as

$$
{ }_{a} I_{t}^{p} f(t)=\frac{1}{\Gamma(p)} \int_{a}^{t} \frac{f(s)}{(t-s)^{1-p}} d s, \quad t>a, p>0,
$$

provided that the right side is point-wise defined on $[a, \infty)$, where $\Gamma(\cdot)$ is the gamma function.

Definition 2 (see [3]). The Riemann-Liouville derivative of order $p>0$ for a function $f:[a, \infty) \rightarrow \mathbb{R}$ is defined as

$$
\begin{array}{r}
{ }_{a}^{L} D_{t}^{p} f(t)=\frac{1}{\Gamma(n-p)} \frac{d^{n}}{d t^{n}} \int_{a}^{t} \frac{f(s)}{(t-s)^{p+1-n}} d s, \\
t>a, n-1<p<n .
\end{array}
$$

Definition 3 (see [3]). The Caputo derivative of order $p>0$ for a function $f:[a, \infty) \rightarrow \mathbb{R}$ is defined as

$$
\begin{array}{r}
{ }_{a}^{C} D_{t}^{p} f(t)={ }_{a}^{L} D_{t}^{p}\left[f(t)-\sum_{k=0}^{n-1} \frac{t^{k}}{k !} f^{(k)}(0)\right], \\
t>a, n-1<p<n .
\end{array}
$$

Let ${ }^{C} D_{t}^{p} f(t)={ }_{0}^{C} D_{t}^{p} f(t)$.
Remark 4. (i) If $f \in C^{n}[a, \infty)$, then

$$
\begin{gathered}
{ }_{a}^{C} D_{t}^{p} f(t)=\frac{1}{\Gamma(n-p)} \int_{a}^{t} \frac{f^{(n)}(s)}{(t-s)^{p+1-n}} d s, \\
t>a, \quad n-1<p<n .
\end{gathered}
$$

(ii) The Caputo derivative of a constant is equal to zero.

(iii)If $f$ is an abstract function with values in $X$, then the integrals which appear in Definitions 1 and 2 are taken in Bochner's sense.

Definition 5. An operator $A$ is said to be sectorial if there are constants $\omega \in \mathbb{R}, \theta \in(0, \pi / 2)$, and $M>0$ such that the resolvent of $A$ exists outside the sector

$$
\Sigma_{\theta}(\omega)=\{\lambda \in \mathbb{C}: \lambda \neq \omega,|\arg (\lambda-\omega)|<\theta\}
$$

with

$$
\|R(\lambda, A)\|_{\mathscr{L}(X)} \leq \frac{M}{|\lambda-\omega|}, \quad \lambda \notin \Sigma_{\theta}(\omega) .
$$

Consider the following Cauchy problem for the Caputo derivative evolution equation of order $\alpha(0<\alpha \leq 1)$ :

$$
\begin{gathered}
{ }^{C} D_{t}^{\alpha} u(t)=A u(t), \quad t \in J=[0, b], \\
u(0)=u_{0} .
\end{gathered}
$$

Definition 6 (see [7]). A family $\left\{S_{\alpha}(t)\right\}_{t \geq 0} \subseteq \mathscr{L}(X)$ is called a solution operator for system (8), if the following conditions are satisfied:

(a) $\left\{S_{\alpha}(t)\right\}_{t \geq 0}$ is strongly continuous, for $t \geq 0$ and $S_{\alpha}(0)=$ $I$.

(b) $S_{\alpha}(t) D(A) \subseteq D(A)$ and $A S_{\alpha}(t) x=S_{\alpha}(t) A x$, for all $x \in D(A)$ and $t \geq 0$.

(c) $S_{\alpha}(t) x$ is a solution of the following integral equation:

$$
u(t)=u_{0}+\frac{1}{\Gamma(\alpha)} \int_{0}^{t} \frac{A u(t)}{(t-s)^{1-\alpha}} d s
$$

for all $x \in D(A)$ and $t \geq 0$.

Remark 7 (see [7]). If $\left\{S_{\alpha}(t)\right\}_{t \geq 0}$ is the solution operator of system (8), then

$$
A x=\Gamma(\alpha+1) \lim _{t \rightarrow 0} \frac{S_{\alpha}(t) x-x}{t^{\alpha}},
$$

where $D(A)$ consists of those $x \in X$ for which this limit exists. We call $A$ the infinitesimal generator of $\left\{S_{\alpha}(t)\right\}_{t \geq 0}$ or say that $A$ generates $\left\{S_{\alpha}(t)\right\}_{t \geq 0}$.

Remark 8 (see [7]). The solution operator $\left\{S_{\alpha}(t)\right\}_{t \geq 0}$ of system (8) is defined as follows:

$$
S_{\alpha}(t)=\frac{1}{2 \pi i} \int_{\gamma} e^{\lambda t} \lambda^{\alpha-1} R\left(\lambda^{\alpha}, A\right) d \lambda,
$$

where $\gamma$ is a suitable path such that $\lambda^{\alpha} \notin \Sigma_{\theta}(\omega)$, for $\lambda \in \gamma$. 
A operator $A$ is said to belong to $\mathfrak{C}^{\alpha}(X, M, \omega)$ or $\mathfrak{C}^{\alpha}(M, \omega)$, if system (8) has solution operator $\left\{S_{\alpha}(t)\right\}_{t \geq 0}$ satisfying $\left|S_{\alpha}(t)\right| \leq M e^{\omega t}, t \geq 0$. Denote $\mathfrak{C}^{\alpha}(\omega)=$ $\bigcup\left\{\mathfrak{C}^{\alpha}(M, \omega), M \geq 1\right\}$ and $\mathfrak{\complement}^{\alpha}=\bigcup\left\{\mathfrak{C}^{\alpha}(\omega), \omega \geq 0\right\}$.

Definition 9 (see [7]). A solution operator $\left\{S_{\alpha}(t)\right\}_{t \geq 0}$ of system (8) is called analytic, if $\left\{S_{\alpha}(t)\right\}_{t \geq 0}$ admits an analytic extension to a sectorial $\sum_{\theta_{0}}=\left\{\lambda \in \mathbb{C} \backslash\{0\}:|\arg \lambda|<\theta_{0}\right\}$ for some $\theta_{0} \in(0, \pi / 2]$. An analytic solution operator is said to be of analyticity type $\left(\theta_{0}, \omega_{0}\right)$, if, for each $\theta<\theta_{0}$ and $\omega>\omega_{0}$, there is an $M=M(\theta, \omega)$ such that $\left|S_{\alpha}(t)\right| \leq e^{\omega R e t}, t \in \sum_{\theta}=\{t \in$ $\mathbb{C} \backslash\{0\}:|\arg t|<\theta\}$. Denote

$$
\begin{aligned}
\mathscr{A}^{\alpha}\left(\theta_{0}, \omega_{0}\right)= & \left\{A \in \mathfrak{C}^{\alpha}: A\right. \text { generates analytic solution } \\
& \left.S_{\alpha}(t) \text { of type }\left(\theta_{0}, \omega_{0}\right)\right\} .
\end{aligned}
$$

Lemma 10 (see [7]). Let $\alpha \in(0,2)$; a linear closed densely defined operator $A$ belongs to $\mathscr{A}^{\alpha}\left(\theta_{0}, \omega_{0}\right)$, if $\lambda^{\alpha} \in \rho(A)$, for each $\lambda \in \Sigma_{\theta_{0}+\pi / 2}$ and, for any $\omega>\omega_{0}, \theta<\theta_{0}$, there is a constant $C=C(\theta, \omega)$ such that

$$
\left\|\lambda^{\alpha-1} R\left(\lambda^{\alpha}, A\right)\right\| \leq \frac{C}{|\lambda-\omega|} \quad \text { for } \lambda \in \Sigma_{\theta+\pi / 2}(\omega) .
$$

Next, we consider the definition of the mild solution of system (1).

According to Definitions 1 and 2, it is suitable to rewrite the nonlocal Cauchy problem (1) in the equivalent integral equation

$$
\begin{aligned}
x(t)= & x_{0}-g(x)+\frac{1}{\Gamma(\alpha)} \\
& \times \int_{0}^{t}(t-s)^{\alpha-1}(A x(s)+f(s, x(s))+B u(s)) d s,
\end{aligned}
$$

provided that the integral in (14) exists.

The following Lemma 11 is discussed in [20]; for the sake of completeness, we outline its proof here.

Lemma 11. If (14) holds and $A$ is a sectorial operator, then we have

$$
\begin{aligned}
x(t)= & S_{\alpha}(t)\left(x_{0}-g(x)\right)+\int_{0}^{t} T_{\alpha}(t-s) f(s, x(s)) d s \\
& +\int_{0}^{t} T_{\alpha}(t-s) B u(s) d s,
\end{aligned}
$$

where

$$
\begin{gathered}
S_{\alpha}(t)=\frac{1}{2 \pi i} \int_{\gamma} e^{\lambda t} \lambda^{\alpha-1} R\left(\lambda^{\alpha}, A\right) d \lambda, \\
T_{\alpha}(t)=\frac{1}{2 \pi i} \int_{\gamma} e^{\lambda t} R\left(\lambda^{\alpha}, A\right) d \lambda,
\end{gathered}
$$

and $\gamma$ is a suitable path such that $\lambda^{\alpha} \notin \Sigma_{\theta}(\omega)$, for $\lambda \in \gamma$.
Proof. By applying the Laplace transform to (14), we have

$$
\mathscr{L}(x)(\lambda)=\frac{x_{0}-g(x)}{\lambda}+\frac{A \mathscr{L}(x)(\lambda)+\mathscr{L}(f+B u)(\lambda)}{\lambda^{\alpha}} .
$$

Since $\left(\lambda^{\alpha} I-A\right)^{-1}$ exists, that is, $\lambda^{\alpha} \in \rho(A)$, from the above equation, we obtain

$$
\begin{aligned}
\mathscr{L}(x)(\lambda)= & \lambda^{\alpha-1}\left(\lambda^{\alpha} I-A\right)^{-1}\left(x_{0}-g(x)\right) \\
& +\left(\lambda^{\alpha} I-A\right)^{-1} \mathscr{L}(f+B u)(\lambda) .
\end{aligned}
$$

Therefore, by the Laplace inverse transform, we have

$$
\begin{aligned}
x(t)= & S_{\alpha}(t)\left(x_{0}-g(x)\right)+\int_{0}^{t} T_{\alpha}(t-s) f(s, x(s)) d s \\
& +\int_{0}^{t} T_{\alpha}(t-s) B u(s) d s .
\end{aligned}
$$

Lemma 12. If $\alpha \in(0,1]$ and $A \in \mathscr{A}^{\alpha}\left(\theta_{0}, \omega_{0}\right)$, then the operators $S_{\alpha}(t)$ and $T_{\alpha}(t)$ are continuous on $t \in \mathbb{R}^{+}$.

Proof. For $0 \leq t^{\prime}<t^{\prime \prime}$, by Lemma 10, we have

$$
\begin{aligned}
\left|S_{\alpha}\left(t^{\prime \prime}\right)-S_{\alpha}\left(t^{\prime}\right)\right| & =\left|\int_{\gamma}\left(e^{\lambda t^{\prime \prime}}-e^{\lambda t^{\prime}}\right) \lambda^{\alpha-1} R\left(\lambda^{\alpha}, A\right) d \lambda\right| \\
& \leq C \int_{\gamma}\left|e^{\lambda t^{\prime \prime}}-e^{\lambda t^{\prime}}\right| \frac{|d \lambda|}{|\lambda-\omega|} ;
\end{aligned}
$$

choose the integration path $\gamma$ as follows:

$$
\begin{gathered}
\gamma=\left\{\omega+r e^{-i((\pi / 2)+\delta)}: \varrho \leq r<\infty\right\} \\
\bigcup\left\{\omega+\varrho e^{i \varphi}:|\varphi| \leq \frac{\pi}{2}+\delta\right\} \\
\bigcup\left\{\omega+r e^{i((\pi / 2)+\delta)}: \varrho \leq r<\infty\right\},
\end{gathered}
$$

such that $\gamma$ is oriented counterclockwise, where $\delta \in\left(0, \delta_{0}\right)$, $\omega>\omega_{0}$, and $\varrho>0$.

From (20), we have

$$
\begin{aligned}
& \left|S_{\alpha}\left(t^{\prime \prime}\right)-S_{\alpha}\left(t^{\prime}\right)\right| \\
& \leq 2 C \int_{\varrho}^{\infty}\left|e^{\lambda t^{\prime \prime}}-e^{\lambda t^{\prime}}\right| \frac{d r}{r}+C \int_{(-\pi / 2)-\delta}^{(\pi / 2)+\delta}\left|e^{\lambda t^{\prime \prime}}-e^{\lambda t^{\prime}}\right| d \varphi \\
& \leq \frac{2 C}{\varrho} \int_{\varrho}^{\infty} \mid e^{(\omega+r \cos ((\pi / 2)+\delta)-i r \sin ((\pi / 2)+\delta)) t^{\prime \prime}} \\
& -e^{(\omega+r \cos ((\pi / 2)+\delta)-i r \sin ((\pi / 2)+\delta)) t^{\prime}} \mid d r \\
& +C \int_{(-\pi / 2)-\delta}^{(\pi / 2)+\delta} \mid e^{(\omega+\varrho \cos \varphi+i \varrho \sin \varphi) t^{\prime \prime}} \\
& -e^{(\omega+\varrho \cos \varphi+i \varrho \sin \varphi) t^{\prime}} \mid d \varphi .
\end{aligned}
$$

By noticing that $\cos ((\pi / 2)+\delta)<0$, by the dominated convergence theorem, we have $\left|S_{\alpha}\left(t^{\prime \prime}\right)-S_{\alpha}\left(t^{\prime}\right)\right| \rightarrow 0$ as $t^{\prime \prime} \rightarrow t^{\prime}$, which implies that $S_{\alpha}(t)$ is continuous on $t$. For the same reason, $T_{\alpha}(t)$ is too continuous on $t$. The proof is complete. 
Lemma 13 (see [20]). If $\alpha \in(0,1]$ and $A \in \mathscr{A}^{\alpha}\left(\theta_{0}, \omega_{0}\right)$, then, for any $t>0$, we have

$$
\left|T_{\alpha}(t)\right| \leq C e^{\omega t}\left(1+t^{\alpha-1}\right), \quad \omega>\omega_{0} .
$$

If $A \in \mathscr{A}^{\alpha}\left(\theta_{0}, \omega_{0}\right)$, then $\left|S_{\alpha}(t)\right| \leq M e^{\omega t}$ and $\left|T_{\alpha}(t)\right| \leq$ $C e^{\omega t}\left(1+t^{\alpha-1}\right)$, for all $t \in(0,+\infty)$. Let

$$
\widetilde{M}_{S}=\sup _{0 \leq t \leq b}\left|S_{\alpha}(t)\right|, \quad \widetilde{M}_{T}=\sup _{0 \leq t \leq b} C e^{\omega t}\left(1+t^{1-\alpha}\right) ;
$$

we have

$$
\left|S_{\alpha}(t)\right| \leq \widetilde{M}_{S}, \quad\left|T_{\alpha}(t)\right| \leq t^{\alpha-1} \widetilde{M}_{T} \quad \forall t \in(0,+\infty) .
$$

Lemma 14 (Krasnoselskii's fixed point theorem). Let $E$ be a Banach space, let $B$ be a bounded closed and convex subset of $E$, and let $F_{1}$ and $F_{2}$ be maps of $B$ into $E$ such that $F_{1} x+F_{2} y \in B$ for every pair $x, y \in B$. If $F_{1}$ is a contraction and $F_{2}$ is completely continuous, then the equation $F_{1} x+F_{2} x=x$ has a solution on $B$.

In [34], Reich gave a general fixed point theorem which contained Krasnoselskii's fixed point theorem, for more details we can see the reference.

Definition 15. A function $x: J \rightarrow X$ is called a mild solution of system (1), if $x$ satisfies the following equation

$$
\begin{aligned}
x(t)= & S_{\alpha}(t)\left(x_{0}-g(x)\right)+\int_{0}^{t} T_{\alpha}(t-s) f(s, x(s)) d s \\
& +\int_{0}^{t} T_{\alpha}(t-s) B u(s) d s,
\end{aligned}
$$

where

$$
\begin{aligned}
& S_{\alpha}(t)=\frac{1}{2 \pi i} \int_{\gamma} e^{\lambda t} \lambda^{\alpha-1} R\left(\lambda^{\alpha}, A\right) d \lambda, \\
& T_{\alpha}(t)=\frac{1}{2 \pi i} \int_{\gamma} e^{\lambda t} R\left(\lambda^{\alpha}, A\right) d \lambda
\end{aligned}
$$

and $\gamma$ is a suitable path such that $\lambda^{\alpha} \notin \Sigma_{\theta}(\omega)$, for $\lambda \in \gamma$.

Remark 16. When $\alpha=1, S_{\alpha}(t)=T_{\alpha}(t)$ is a $C_{0}$-semigroup and system (1) degenerates into 1 order evolution equation. However, the limits of $S_{\alpha}(t)$ and $T_{\alpha}(t)$ in [21-23] did not exist as $\alpha \rightarrow 1^{-}$.

Remark 17. When $A$ generates a $C_{0}$-semigroup $\{T(t)\}_{t>0}$ in system (1), we have

$$
\begin{gathered}
S_{\alpha}(t)=\int_{0}^{\infty} \phi_{\alpha}(\theta) T\left(t^{\alpha} \theta\right) d \theta, \\
T_{\alpha}(t)=\alpha t^{\alpha-1} \int_{0}^{\infty} \theta \phi_{\alpha}(\theta) T\left(t^{\alpha} \theta\right) d \theta,
\end{gathered}
$$

where $0<\alpha<1$ and $\phi(\theta)$ is a probability density function defined on $(0, \infty)$ in [21-23]. So, this definition is more general to that in [21-23].

Remark 18. It is easy to verify that a classical solution of system (1) is a mild solution of the same system.
Definition 19. The system (1) is said to be completely controllable on $J$, if, for every $x_{0}, x_{1} \in X$, there exists a control $u \in L^{2}(J, U)$, such that a mild solution $x$ of system (1) satisfies $x(b)=x_{1}$.

In this paper, we assume the following.

$\left(\mathrm{H}_{1}\right) f: J \times X \rightarrow X$ is continuous and there exist constant $q \in(0, \alpha)$ and function $m \in L^{1 / q}\left(J, \mathbb{R}^{+}\right)$such that

$$
|f(t, x(t))-f(t, y(t))| \leq m(t)\|x-y\|,
$$

for all $t \in J$ and $x, y \in X$.

$\left(\mathrm{H}_{2}\right) g: X \rightarrow X$ is continuous and there exists a constant $L_{g}>0$ such that

$$
\|g(x)-g(y)\| \leq L_{g}\|x-y\|,
$$

for all $x, y \in X$.

It is easy to see that if $\left(\mathrm{H}_{2}\right)$ holds, then the following assumption holds:

$\left(\mathrm{H}_{2}^{\prime}\right) g: X \rightarrow X$ is continuous and there exist positive constants $K_{g}$ and $d$ such that

$$
\|g(x)\| \leq K_{g}\|x\|+d,
$$

for all $x \in X$;

$\left(\mathrm{H}_{3}\right)$ the operator family $\left\{S_{\alpha}(t)\right\}_{t \geq 0}$ is compact;

$\left(\mathrm{H}_{4}\right)$ the linear operator $B: L^{2}(J, U) \rightarrow L(J, X)$ is bounded; $W: L^{2}(J, U) \rightarrow X$ defined by

$$
W u=\int_{0}^{b} T_{\alpha}(b-s) B u(s) d s
$$

has an inverse operator $W^{-1}$ which takes values in $L^{2}(J, U) / \operatorname{Ker} W$ and there exist two positive constants $M_{2}, M_{3}>0$ such that

$$
\|B\| \leq M_{2}, \quad\left\|W^{-1}\right\| \leq M_{3} .
$$

\section{Complete Controllability Results}

Theorem 20. Suppose that $\left(H_{1}\right),\left(H_{2}\right)$, and $\left(H_{4}\right)$ are satisfied; then system (1) is completely controllable on $J$, provided that $A \in \mathscr{A}^{\alpha}\left(\theta_{0}, \omega_{0}\right)$ and

$$
\begin{aligned}
\Theta= & \left(\widetilde{M}_{S} L_{g}+\widetilde{M}_{T} b^{\alpha-q}\left(\frac{1-q}{\alpha-q}\right)^{1-q}\|m\|_{L^{1 / q}}\right) \\
& \times\left(1+M_{2} M_{3} \widetilde{M}_{T} \frac{b^{\alpha}}{\alpha}\right)<1 .
\end{aligned}
$$

Proof. Using hypothesis $\left(\mathrm{H}_{4}\right)$ for an arbitrary function $x \in$ $C(J, X)$, we defined the control function $u_{x}(t)$ by

$$
\begin{aligned}
u_{x}(t)=W^{-1} & \left(x_{1}-S_{\alpha}(b)\left(x_{0}-g(x)\right)\right. \\
& \left.\quad-\int_{0}^{b} T_{\alpha}(b-s) f(s, x(s)) d s\right) .
\end{aligned}
$$


We show that using this control, the operator $F$ on $C(J, X)$ by

$$
\begin{aligned}
(F x)(t)= & S_{\alpha}(t)\left(x_{0}-g(x)\right)+\int_{0}^{t} T_{\alpha}(t-s) f(s, x(s)) d s \\
& +\int_{0}^{t} T_{\alpha}(t-s) B u_{x}(s) d s
\end{aligned}
$$

has a fixed point $x$, which is a mild solution of system (1).

It is obvious that $(F x)(b)=x_{1}$, which means that $u_{x}$ steers the mild $x$ from $x_{0}$ to $x_{1}$ in finite time $b$. This implies that system (1) is completely controllable on $J$. Next, we will prove that $F$ has a fixed point on $C(J, X)$.

Taking $t \in[0, b]$ and, for all $x, y \in C(J, X)$, we have, from $\left(\mathrm{H}_{1}\right),\left(\mathrm{H}_{4}\right)$, and (35),

$$
\begin{aligned}
& \left|u_{x}(t)-u_{y}(t)\right| \\
& =\| W^{-1}\left(S_{\alpha}(b)(g(x)-g(y))\right. \\
& \left.+\int_{0}^{b} T_{\alpha}(b-s) f((s, x(s))-f(s, y(s))) d s\right) \| \\
& \leq M_{3} \widetilde{M}_{T} \int_{0}^{b}(b-s)^{\alpha-1} m(s) d s\|x-y\| \\
& +M_{3} \widetilde{M}_{S} L_{g}\|x-y\| \\
& \leq\left(M_{3} \widetilde{M}_{T} b^{\alpha-q}\left(\frac{1-q}{\alpha-q}\right)^{1-q}\|m\|_{L^{1 / q}}+M_{3} \widetilde{M}_{S} L_{g}\right)\|x-y\| ;
\end{aligned}
$$

by (25), we have

$$
\begin{aligned}
|(F x)(t)-(F y)(t)| & \mid S_{\alpha}(t)(g(y)-g(x)) \\
& +\int_{0}^{t} T_{\alpha}(t-s)(f(s, x(s))-f(s, y(s))) d s \\
& +\int_{0}^{t} T_{\alpha}(t-s) B\left(u_{x}(s)-u_{y}(s)\right) d s \mid \\
\leq & \left|S_{\alpha}(t)\right|\|g(y)-g(x)\| \\
& +\left|\int_{0}^{t} T_{\alpha}(t-s)(f(s, x(s))-f(s, y(s))) d s\right| \\
& +\left|\int_{0}^{t} T_{\alpha}(t-s) B\left(u_{x}(s)-u_{y}(s)\right) d s\right| \\
\leq & \widetilde{M}_{S} L_{g}\|y-x\|+\widetilde{M}_{T} \int_{0}^{t}(t-s)^{\alpha-1} m(s)\|y-x\| d s \\
& +\widetilde{M}_{T} \int_{0}^{t}(t-s)^{\alpha-1}\left|B\left(u_{x}(s)-u_{y}(s)\right)\right| d s \\
\leq & \widetilde{M}_{S} L_{g}\|y-x\|+\widetilde{M}_{T}\left(\int_{0}^{t}(t-s)^{(\alpha-1) /(1-q)} d s\right)^{1-q} \\
\times & \left(\int_{0}^{t}(m(s))^{1 / q} d s\right)^{q}\|y-x\|+\frac{b^{\alpha}}{\alpha} M_{2} M_{3} \widetilde{M}_{T} \\
\times & \left(\widetilde{M}_{T} \frac{b^{2 \alpha-q}}{\alpha}\left(\frac{1-q}{\alpha-q}\right)^{1-q}\|m\|_{L^{1 / q}}+\widetilde{M}_{S} L_{g}\right)\|y-x\|
\end{aligned}
$$

$$
\begin{aligned}
& \leq\left[\widetilde{M}_{S} L_{g}+\widetilde{M}_{T} b^{\alpha-q}\left(\frac{1-q}{\alpha-q}\right)^{1-q}\|m\|_{L^{1 / q}}\right. \\
& \left.\quad \times\left(1+M_{2} M_{3} \widetilde{M}_{T} \frac{b^{\alpha}}{\alpha}\right)\right]\|y-x\|=\Theta\|y-x\| .
\end{aligned}
$$

Hence, $F$ is a contraction mapping and has a unique fixed point $x^{*} \in C(J, X)$. Therefore, this $x^{*}$ is a mild solution of system (1). The proof is complete.

Theorem 21. Suppose that $\left(H_{1}\right),\left(H_{2}^{\prime}\right),\left(H_{3}\right)$, and $\left(H_{4}\right)$ are satisfied; then system (1) is completely controllable on J provided that $A \in \mathscr{A}^{\alpha}\left(\theta_{0}, \omega_{0}\right)$ and

$$
\begin{aligned}
M_{4}= & \left(M_{2} M_{3} \widetilde{M}_{T} \frac{b^{\alpha}}{\alpha}+1\right) \\
& \times\left[\widetilde{M}_{S} K_{g}+\widetilde{M}_{T}\left(\frac{1-q}{\alpha-q}\right)^{1-q} b^{\alpha-q}\|m\|_{L^{1 / q}}\right]<1 .
\end{aligned}
$$

Proof. Define

$$
\begin{aligned}
\left(F_{1} x\right)(t)= & \int_{0}^{t} T_{\alpha}(t-s) f(s, x(s)) d s \\
& +\int_{0}^{t} T_{\alpha}(t-s) B u_{x}(s) d s, \\
\left(F_{2} x\right)(t)= & S_{\alpha}(t)\left(x_{0}-g(x)\right),
\end{aligned}
$$

for $t \in[0, b]$ and any $x \in C(J, X)$. Taking into account (35), by $\left(\mathrm{H}_{1}\right),\left(\mathrm{H}_{2}^{\prime}\right)$, and $\left(\mathrm{H}_{4}\right)$, we have

$$
\begin{aligned}
& \left|u_{x}(t)\right|=\| W^{-1}\left(x_{1}-S_{\alpha}(b)\left(x_{0}-g(x)\right)\right. \\
& \left.-\int_{0}^{b} T_{\alpha}(b-s) f(s, x(s)) d s\right) \| \\
& \leq\left\|W^{-1}\right\|\left(\left\|x_{1}\right\|+\left|S_{\alpha}(b)\left(x_{0}-g(x)\right)\right|\right. \\
& \left.+\left|\int_{0}^{b} T_{\alpha}(b-s) f(s, x(s)) d s\right|\right) \\
& \leq M_{3}\left(\left\|x_{1}\right\|+\widetilde{M}_{S}\left\|x_{0}-g(x)\right\|\right. \\
& \left.+\widetilde{M}_{T} \int_{0}^{b}(b-s)^{\alpha-1}(m(s)\|x\|+|f(s, 0)|) d s\right) \\
& \leq M_{3}\left[\left\|x_{1}\right\|+\widetilde{M}_{S}\left(\left\|x_{0}\right\|+K_{g}\|x\|+d\right)\right. \\
& +\widetilde{M}_{T}\left(\left(\frac{1-q}{\alpha-q}\right)^{1-q} b^{\alpha-q}\|m\|_{L^{1 / q}}\right. \\
& \left.\left.\times\|x\|+\frac{b^{\alpha}}{\alpha} \sup _{s \in J}|f(s, 0)|\right)\right] .
\end{aligned}
$$


In order to make the following process clear, we divide it into several steps.

Step I. For $t \in[0, b]$ and any $x, y \in C(J, X)$, we have

$$
\begin{aligned}
& \left|\left(F_{1} x\right)(t)\right| \\
& =\mid \int_{0}^{t} T_{\alpha}(t-s) f(s, x(s)) d s \\
& +\int_{0}^{t} T_{\alpha}(t-s) B u_{x}(s) d s \\
& \leq\left|\int_{0}^{t} T_{\alpha}(t-s)(f(s, x(s))-f(s, 0)) d s\right| \\
& +\left|\int_{0}^{t} T_{\alpha}(t-s) f(s, 0) d s\right|+\left|\int_{0}^{t} T_{\alpha}(t-s) B u_{x}(s) d s\right| \\
& \leq \widetilde{M}_{T} \int_{0}^{t}(t-s)^{\alpha-1} m(s)\|x\| d s \\
& +\widetilde{M}_{T} \sup _{s \in J}|f(s, 0)| \int_{0}^{t}(t-s)^{\alpha-1} d s \\
& +\widetilde{M}_{T} \int_{0}^{t}(t-s)^{\alpha-1} M_{2}\left\|u_{x}(s)\right\| d s \\
& \leq \widetilde{M}_{T}\left(\int_{0}^{t}(t-s)^{(\alpha-1) /(1-q)} d s\right)^{1-q}\|m\|_{L^{1 / q}}\|x\| \\
& +\frac{b^{\alpha}}{\alpha} \widetilde{M}_{T} \sup _{s \in[0, b]}|f(s, 0)|+\frac{b^{\alpha}}{\alpha} \\
& \times \widetilde{M}_{T} M_{2} M_{3}\left[\left\|x_{1}\right\|+\widetilde{M}_{S}\left(\left\|x_{0}\right\|+K_{g}\|x\|+d\right)\right. \\
& +\widetilde{M}_{T}\left(\left(\frac{1-q}{\alpha-q}\right)^{1-q} b^{\alpha-q}\|m\|_{L^{1 / q}}\|x\|\right. \\
& \left.\left.+\frac{b^{\alpha}}{\alpha} \sup _{s \in J}|f(s, 0)|\right)\right] \\
& \leq\left[\left(1+\frac{b^{\alpha}}{\alpha} \widetilde{M}_{T} M_{2} M_{3}\right) \widetilde{M}_{T}\left(\frac{1-q}{\alpha-q}\right)^{1-q} b^{\alpha-q}\|m\|_{L^{1 / q}}\right. \\
& \left.+M_{2} M_{3} \widetilde{M}_{T} \frac{b^{\alpha}}{\alpha} \widetilde{M}_{S} K_{g}\right]\|x\| \\
& +M_{2} M_{3} \widetilde{M}_{T} \frac{b^{\alpha}}{\alpha}\left[\left\|x_{1}\right\|+\widetilde{M}_{S}\left(\left\|x_{0}\right\|+d\right)\right] \\
& +\left(M_{2} M_{3} \widetilde{M}_{T} \frac{b^{\alpha}}{\alpha}+1\right) \frac{b^{\alpha}}{\alpha} \widetilde{M}_{T} \sup _{s \in J}|f(s, 0)|, \\
& \left|\left(F_{2} y\right)(t)\right|=\left|S_{\alpha}(t)\left(x_{0}-g(y)\right)\right| \\
& \leq \widetilde{M}_{S}\left(\left\|x_{0}\right\|+K_{g}\|y\|+d\right) \text {. }
\end{aligned}
$$

By the condition $M_{4}<1$, we can find $k_{0}>0$ such that, for $x, y \in B_{k_{0}}=\left\{x \in C(J, X):\|x\| \leq k_{0}\right\}$,

$$
\left\|F_{1} x+F_{2} y\right\| \leq k_{0} \text { i.e. } F_{1} x+F_{2} y \in B_{k_{0}} .
$$

Step II. $F_{1}$ is a contraction mapping on $B_{k_{0}}$.
For any $x, y \in B_{k_{0}}$ and $t \in[0, b]$, we have

$$
\begin{aligned}
\left|\left(F_{1} x\right)(t)-\left(F_{1} y\right)(t)\right| & \left|\int_{0}^{t} T_{\alpha}(t-s)(f(s, x(s))-f(s, y(s))) d s\right| \\
& +\left|\int_{0}^{t} T_{\alpha}(t-s) B\left(u_{x}(s)-u_{y}(s)\right) d s\right| \\
\leq & \widetilde{M}_{T} \int_{0}^{t}(t-s)^{\alpha-1} m(s)\|x-y\| d s \\
& +\widetilde{M}_{T} M_{2} \int_{0}^{t}(t-s)^{\alpha-1}\left|u_{x}(s)-u_{y}(s)\right| d s \\
\leq & \widetilde{M}_{T}\left(\int_{0}^{t}(t-s)^{(\alpha-1) /(1-q)} d s\right)^{1-q}\|m\|_{L^{1 / q}}\|x-y\| \\
& +M_{2} \widetilde{M}_{T} \frac{b^{\alpha}}{\alpha}\left(M_{3} \widetilde{M}_{T} b^{\alpha-q}\left(\frac{1-q}{\alpha-q}\right)^{1-q}\|m\|_{L^{1 / q}}\right. \\
\leq & +\widetilde{M}_{T}\left(\frac{1-q}{\alpha-q}\right)^{1-q} b^{\alpha-q}\|m\|_{L^{1 / q}}\left(1+\frac{b^{\alpha}}{\alpha} \widetilde{M}_{T} M_{2} M_{3}\right) \\
& \left.+\frac{b^{\alpha}}{\alpha} \widetilde{M}_{T} M_{2} M_{3} \widetilde{M}_{S} K_{g}\right)\|x-y\|=: \Upsilon\|x-y\| .
\end{aligned}
$$

From the condition $M_{4}<1$, we obtain $\Upsilon<1$, which implies that $F_{1}$ is a contraction mapping.

Step III. $F_{2}$ is a completely continuous operator.

First, we will prove that $F_{2}$ is continuous on $B_{k_{0}}$. Let $\left\{x_{n}\right\} \subseteq$ $B_{k_{0}}$ with $x_{n} \rightarrow x \in B_{k_{0}}$. By $\left(\mathrm{H}_{2}^{\prime}\right)$, we have

$$
g\left(x_{n}\right) \longrightarrow g(x) \text { as } n \longrightarrow \infty \text {. }
$$

So, we have

$$
\begin{aligned}
& \left|\left(F_{2} x_{n}\right)(t)-\left(F_{2} x\right)(t)\right| \\
& \quad=\left|S_{\alpha}(t)\left(x_{0}-g\left(x_{n}\right)\right)-S_{\alpha}(t)\left(x_{0}-g(x)\right)\right| \\
& \quad=\left|S_{\alpha}(t)\left(g(x)-g\left(x_{n}\right)\right)\right| \\
& \quad \leq \widetilde{M}_{S}\left\|g(x)-g\left(x_{n}\right)\right\| \longrightarrow 0 \text { as } n \longrightarrow \infty,
\end{aligned}
$$

which implies that $F_{2}$ is continuous.

Next, we will show that $\left\{F_{2} x, x \in B_{k_{0}}\right\}$ is relatively compact. It suffices to show that the family of function $\left\{F_{2} x, x \in B_{k_{0}}\right\}$ is uniformly bounded and equicontinuous and, for any $t \in[0, b],\left\{\left(F_{2} x\right)(t), x \in B_{k_{0}}\right\}$ is relatively compact.

For any $x \in B_{k_{0}}$, we have $\left\|F_{2} x\right\| \leq k_{0}$ which implies that $\left\{F_{2} x, x \in B_{k_{0}}\right\}$ is uniformly bounded. In the following, we will show that $\left\{F_{2} x, x \in B_{k_{0}}\right\}$ is a family of equicontinuous functions.

For any $x \in B_{k_{0}}$ and $0 \leq t^{\prime}<t^{\prime \prime} \leq b$, we have

$$
\begin{aligned}
& \left|\left(F_{2} x\right)\left(t^{\prime \prime}\right)-\left(F_{2} x\right)\left(t^{\prime}\right)\right| \\
& \quad=\left|S_{\alpha}\left(t^{\prime \prime}\right)\left(x_{0}-g(x)\right)-S_{\alpha}\left(t^{\prime}\right)\left(x_{0}-g(x)\right)\right| \\
& \quad \leq\left|S_{\alpha}\left(t^{\prime \prime}\right)-S_{\alpha}\left(t^{\prime}\right)\right|\left\|x_{0}-g(x)\right\| \\
& \quad \leq\left|S_{\alpha}\left(t^{\prime \prime}\right)-S_{\alpha}\left(t^{\prime}\right)\right|\left(\left\|x_{0}\right\|+K_{g} k_{0}+d\right) .
\end{aligned}
$$


From Lemma 12, we have $\left|\left(F_{2} x\right)\left(t^{\prime \prime}\right)-\left(F_{2} x\right)\left(t^{\prime}\right)\right| \rightarrow 0$ independently of $x \in B_{k_{0}}$ as $t^{\prime \prime}-t^{\prime} \rightarrow 0$, which means that $\left\{F_{2} x, x \in\right.$ $\left.B_{k_{0}}\right\}$ is equicontinuous.

By the compactness of $\left\{S_{\alpha}(t)\right\}_{t \geq 0}$, we know that $\left\{\left(F_{2} x\right)(t)\right.$, $\left.x \in B_{k_{0}}\right\}$ is relatively compact. Therefore, $\left\{F_{2} x, x \in B_{k_{0}}\right\}$ is relatively compact by Arzela-Ascoli theorem. The continuity of $F_{2}$ and relative compactness of $\left\{F_{2} x, x \in B_{k_{0}}\right\}$ imply that $F_{2}$ is a completely continuous operator. By using Krasnoselskii's fixed point theorem, we obtain that $F_{1}+F_{2}$ has a fixed point on $B_{k_{0}}$. Therefore, the nonlocal Cauchy problem (1) has at least one mild solution. The proof is complete.

When there is no control term, system (1) degenerates to the following system:

$$
\begin{gathered}
{ }^{C} D_{t}^{\alpha} x(t)=A x(t)+f(t, x(t)), \quad t \in J=[0, b], \\
x(0)+g(x)=x_{0} .
\end{gathered}
$$

As a direct result of Theorems 20 and 21, we have the following corollaries.

Corollary 22. Suppose that $\left(H_{1}\right),\left(H_{2}\right)$, and $\left(H_{4}\right)$ are satisfied; then system (48) has a unique mild solution, if $A \in \mathscr{A}^{\alpha}\left(\theta_{0}, \omega_{0}\right)$ and

$$
\Theta=\widetilde{M}_{S} L_{g}+\widetilde{M}_{T} b^{\alpha-q}\left(\frac{1-q}{\alpha-q}\right)^{1-q}\|m\|_{L^{1 / q}}<1 .
$$

Corollary 23. Suppose that $\left(H_{1}\right),\left(H_{2}^{\prime}\right),\left(H_{3}\right)$, and $\left(H_{4}\right)$ are satisfied; then system (48) has at least one mild solution, if $A \in \mathscr{A}^{\alpha}\left(\theta_{0}, \omega_{0}\right)$ and

$$
M_{4}=\widetilde{M}_{S} K_{g}+\widetilde{M}_{T}\left(\frac{1-q}{\alpha-q}\right)^{1-q} b^{\alpha-q}\|m\|_{L^{1 / q}}<1 .
$$

\section{Conclusions}

In this paper, we introduce a more general definition for mild solution of fractional evolution equation with nonlocal condition based on solution operator. By contraction fixed point theorem and Krasnoselskii's fixed point theorem, we obtain some sufficient conditions to ensure the complete controllability for system (1). Here, we do not require the operator $A$ to be the infinitesimal generator of an analytic semigroup $\{T(t)\}_{t \geq 0}$ of uniform boundedness. So, the results we obtained are more general. For fractional evolution equation with Riemann-Liouville derivative, since it is equipped with a singular initial, it will be a difficult problem.

\section{Conflict of Interests}

The authors declare that there is no conflict of interests regarding the publishing of this paper.

\section{Acknowledgments}

The authors would like to thank the Editor and the anonymous reviewers for their helpful comments and suggestions. This work was supported by the National Natural Science
Foundation of China (11271309 and 11301451), the Specialized Research Fund for the Doctoral Program of Higher Education (20114301110001), and the Key Projects of Hunan Provincial Natural Science Foundation of China (12JJ2001).

\section{References}

[1] A. A. Kilbas, H. M. Srivastava, and J. J. Trujillo, "Theory an application of fractional differential equations," in North-Holland Mathematics Studies, vol. 204, Elsevier Science B.V., Amsterdam, The Netherlands, 2006.

[2] K. S. Miller and B. Ross, An Introduction to the Fractional Calculus and Differential Equations, John Wiley \& Sons, New York, NY, USA, 1993.

[3] I. Podlubny, Fractional Differential Equations, vol. 198, Academic Press, San Diego, Calif, USA, 1999.

[4] V. Lakshmikantham, S. Leela, and J. V. Devi, Theory of Fractional Dynamic Systems, Cambridge Scientific, Cambridge, UK, 2009.

[5] R. P. Agarwal, V. Lakshmikantham, and J. J. Nieto, "On the concept of solution for fractional differential equations with uncertainty," Nonlinear Analysis. Theory, Methods \& Applications A: Theory and Methods, vol. 72, no. 6, pp. 2859-2862, 2010.

[6] Z. B. Bai and Y. H. Zhang, "Solvability of fractional threepoint boundary value problems with nonlinear growth," Applied Mathematics and Computation, vol. 218, no. 5, pp. 1719-1725, 2011.

[7] E. Bazhlekova, Fractional evolution equations in Banach space [Ph.D. thesis], Eindhoven University of Technology, Eindhoven, The Netherlands, 2001.

[8] M. Benchohra and A. Ouahab, "Controllability results for functional semilinear differential inclusions in Fréchet spaces," Nonlinear Analysis. Theory, Methods \& Applications A: Theory and Methods, vol. 61, no. 3, pp. 405-423, 2005.

[9] F. L. Chen, J. J. Nieto, and Y. Zhou, "Global attractivity for nonlinear fractional differential equations," Nonlinear Analysis. Real World Applications, vol. 13, no. 1, pp. 287-298, 2012.

[10] S. D. Eidelman and A. N. Kochubei, "Cauchy problem for fractional diffusion equations," Journal of Differential Equations, vol. 199, no. 2, pp. 211-255, 2004.

[11] V. Lakshmikantham and A. S. Vatsala, "Basic theory of fractional differential equations," Nonlinear Analysis. Theory, Methods \& Applications A: Theory and Methods, vol. 69, no. 8, pp. 2677-2682, 2008.

[12] J. P. Yan and C. P. Li, "On chaos synchronization of fractional differential equations," Chaos, Solitons \& Fractals, vol. 32, no. 2, pp. 725-735, 2007.

[13] Y. Li, Y. Chen, and I. Podlubny, "Stability of fractional-order nonlinear dynamic systems: Lyapunov direct method and generalized Mittag-Leffler stability," Computers \& Mathematics with Applications, vol. 59, no. 5, pp. 1810-1821, 2010.

[14] H. P. Ye, J. M. Gao, and Y. S. Ding, "A generalized Gronwall inequality and its application to a fractional differential equation," Journal of Mathematical Analysis and Applications, vol. 328, no. 2, pp. 1075-1081, 2007.

[15] R. P. Agarwal, M. Belmekki, and M. Benchohra, "A survey on semilinear differential equations and inclusions involving Riemann-Liouville fractional derivative," Advances in Difference Equations, vol. 2009, Article ID 981728, 47 pages, 2009.

[16] M. Belmekki and M. Benchohra, "Existence results for fractional order semilinear functional differential equations with 
nondense domain," Nonlinear Analysis. Theory, Methods \& Applications A: Theory and Methods, vol. 72, no. 2, pp. 925-932, 2010.

[17] E. Hernández, D. O’Regan, and K. Balachandran, “On recent developments in the theory of abstract differential equations with fractional derivatives," Nonlinear Analysis. Theory, Methods \& Applications A: Theory and Methods, vol. 73, no. 10, pp. 34623471, 2010.

[18] L. Hu, Y. Ren, and R. Sakthivel, "Existence and uniqueness of mild solutions for semilinear integro-differential equations of fractional order with nonlocal initial conditions and delays," Semigroup Forum, vol. 79, no. 3, pp. 507-514, 2009.

[19] G. M. Mophou, "Existence and uniqueness of mild solutions to impulsive fractional differential equations," Nonlinear Analysis. Theory, Methods \& Applications A: Theory and Methods, vol. 72, no. 3-4, pp. 1604-1615, 2010.

[20] X.-B. Shu, Y. Lai, and Y. Chen, "The existence of mild solutions for impulsive fractional partial differential equations," Nonlinear Analysis. Theory, Methods \& Applications A: Theory and Methods, vol. 74, no. 5, pp. 2003-2011, 2011.

[21] J. R. Wang and Y. Zhou, "Complete controllability of fractional evolution systems," Communications in Nonlinear Science and Numerical Simulation, vol. 17, no. 11, pp. 4346-4355, 2012.

[22] Y. Zhou and F. Jiao, "Nonlocal Cauchy problem for fractional evolution equations," Nonlinear Analysis. Real World Applications, vol. 11, no. 5, pp. 4465-4475, 2010.

[23] Y. Zhou and F. Jiao, "Existence of mild solutions for fractional neutral evolution equations," Computers \& Mathematics with Applications, vol. 59, no. 3, pp. 1063-1077, 2010.

[24] M. Fečkan, Y. Zhou, and J. Wang, "On the concept and existence of solution for impulsive fractional differential equations," Communications in Nonlinear Science and Numerical Simulation, vol. 17, no. 7, pp. 3050-3060, 2012.

[25] X.-B. Shu and Q. Wang, "The existence and uniqueness of mild solutions for fractional differential equations with nonlocal conditions of order $1<\alpha<2$," Computers \& Mathematics with Applications, vol. 64, no. 6, pp. 2100-2110, 2012.

[26] R. P. Agarwal, B. Ahmad, A. Alsaedi, and N. Shahzad, "Existence and dimension of the set of mild solutions to semilinear fractional differential inclusions," Advances in Difference Equations, vol. 2012, p. 74, 2012.

[27] N. U. Ahmed, Dynamic Systems and Control with Applications, World Scientific, Hackensack, NJ, USA, 2006.

[28] K. Balachandran and R. Sakthivel, "Controllability of functional semilinear integrodifferential systems in Banach spaces," Journal of Mathematical Analysis and Applications, vol. 255, no. 2, pp. 447-457, 2001.

[29] Y. K. Chang, J. J. Nieto, and W. S. Li, "Controllability of semilinear differential systems with nonlocal initial conditions in Banach spaces," Journal of Optimization Theory and Applications, vol. 142, no. 2, pp. 267-273, 2009.

[30] Z. Tai and X. Wang, "Controllability of fractional-order impulsive neutral functional infinite delay integrodifferential systems in Banach spaces," Applied Mathematics Letters, vol. 22, no. 11, pp. 1760-1765, 2009.

[31] Z. Yan, "Controllability of fractional-order partial neutral functional integrodifferential inclusions with infinite delay," Journal of the Franklin Institute. Engineering and Applied Mathematics, vol. 348, no. 8, pp. 2156-2173, 2011.

[32] L. Byszewski, "Theorems about the existence and uniqueness of solutions of a semilinear evolution nonlocal Cauchy problem,"
Journal of Mathematical Analysis and Applications, vol. 162, no. 2, pp. 494-505, 1991.

[33] L. Byszewski and V. Lakshmikantham, "Theorem about the existence and uniqueness of a solution of a nonlocal abstract Cauchy problem in a Banach space," Applicable Analysis, vol. 40, no. 1, pp. 11-19, 1991.

[34] S. Reich, "Fixed points of condensing functions," Journal of Mathematical Analysis and Applications, vol. 41, pp. 460-467, 1973. 


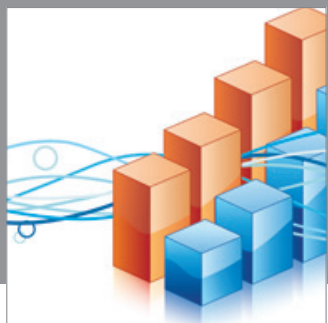

Advances in

Operations Research

mansans

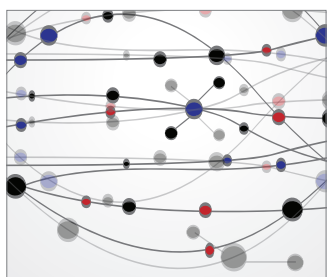

The Scientific World Journal
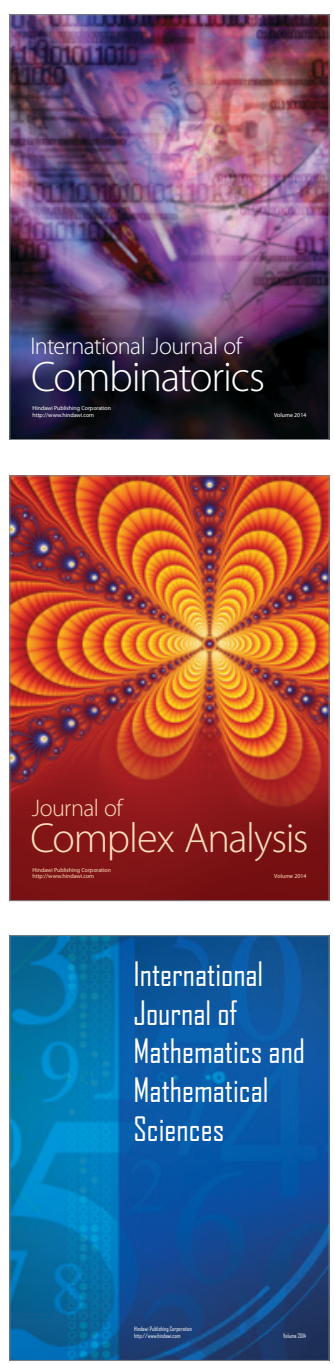
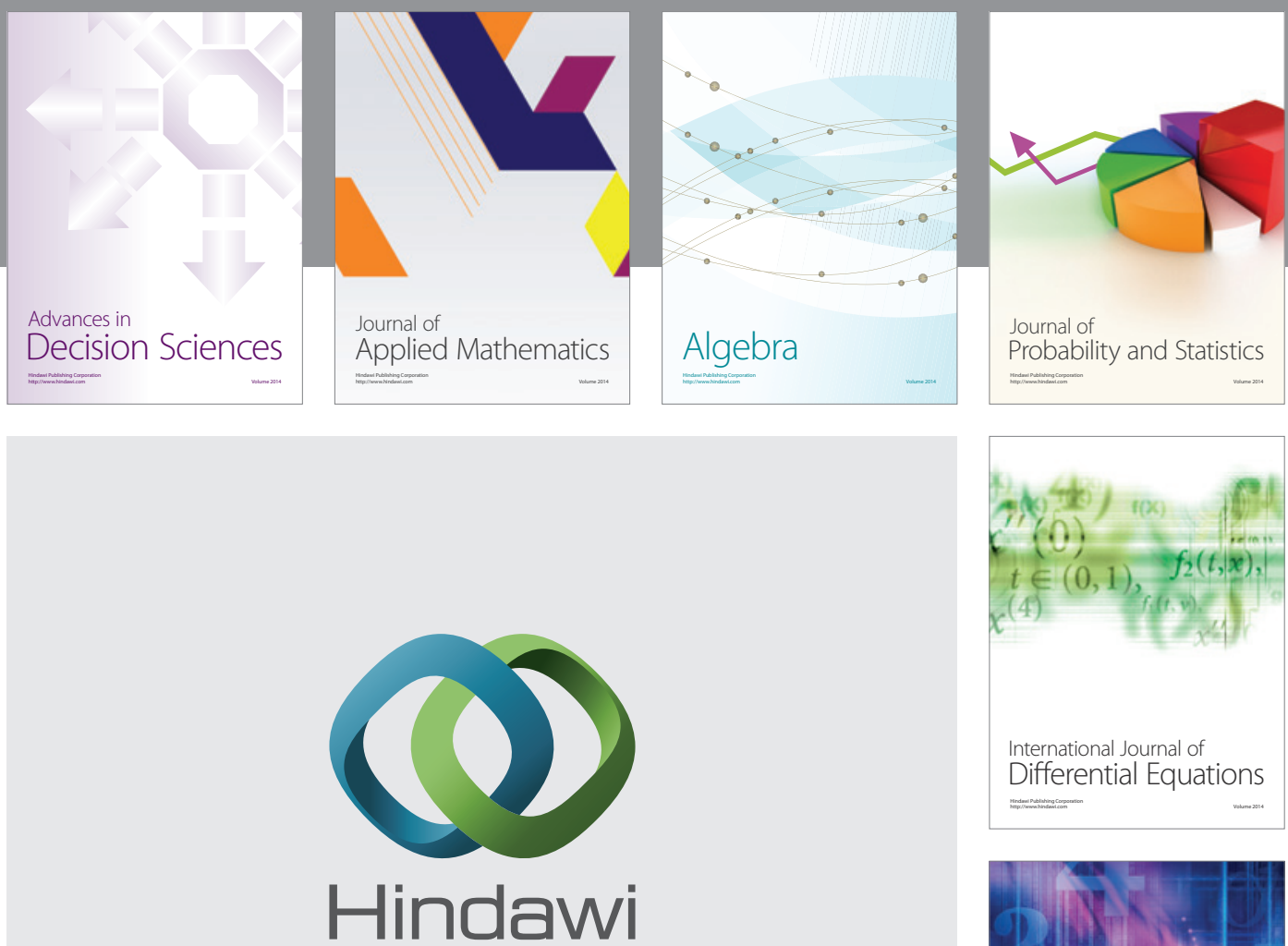

Submit your manuscripts at http://www.hindawi.com
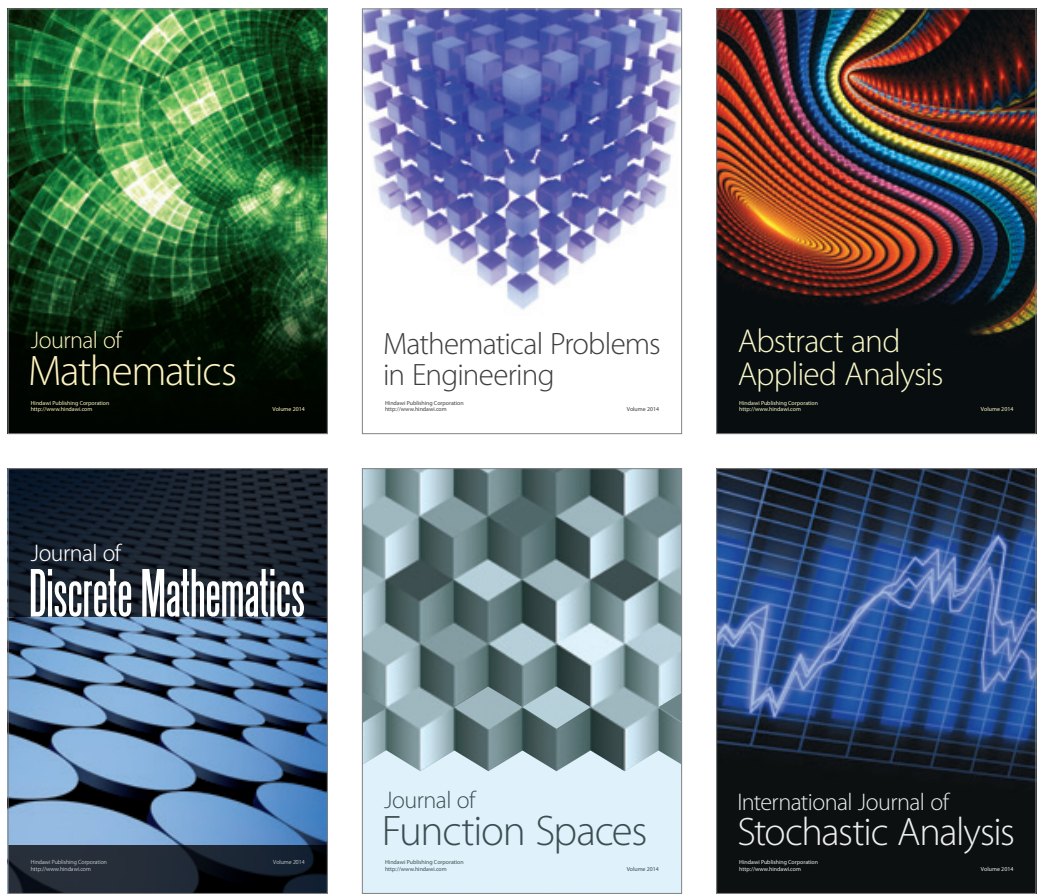

Journal of

Function Spaces

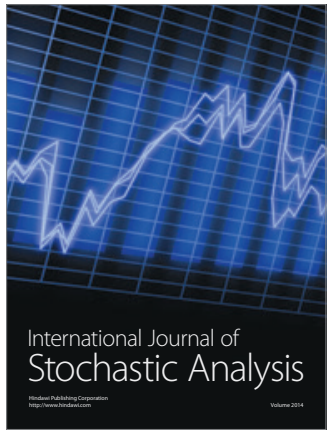

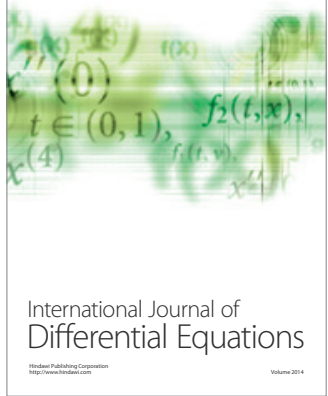
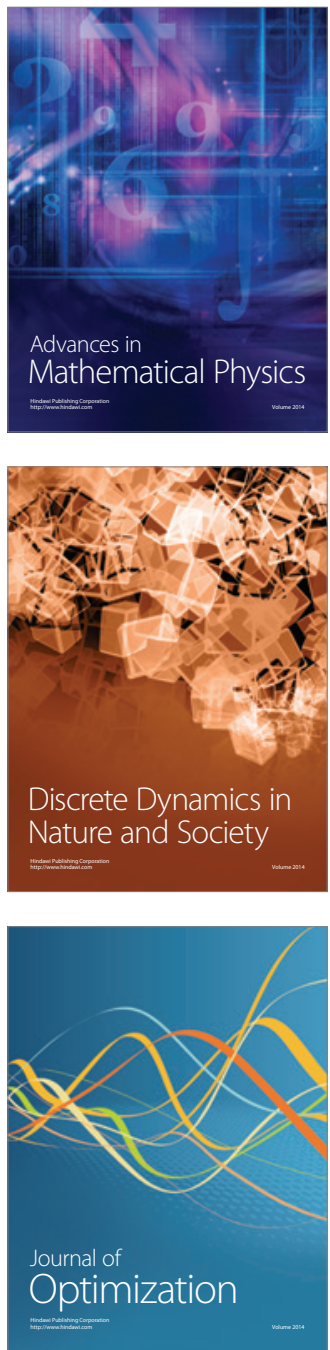